\title{
A SUBSEQUENCE PRINCIPLE IN PROBABILITY THEORY (APPLIED TO THE LAW OF THE ITERATED LOGARITHM)
}

\author{
BY S. D. CHATTERJI
}

Communicated by Anatole Beck, October 8, 1973

It is a remarkable fact that the classical limit laws of probability theory concerning independent identically distributed random variables remain valid, with only minor modifications, for sequences of random variables which are quite far from being independent. This phenomenon has induced me in recent years to formulate the following heuristic principle of subsequences ([2], [3], [4]): let $\Pi$ be a quantitative asymptotic property valid for any sequence of independent identically distributed random variables $X_{n}$ belonging to some integrability class defined by a norm $\|\cdot\|_{L} ;$ then an analogous property $\tilde{\Pi}$ is valid for a suitable subsequence $\left\{f_{n(k)}\right\}$ of any sequence of random variables $\left\{f_{n}\right\}$ if only $\sup _{n}\left\|f_{n}\right\|_{L}<\infty$. Moreover, the subsequence can be chosen in such a way that any further subsequence of it will have the same property $\tilde{\Pi}$.

When $\Pi$ is the Kolmogorov strong law of large numbers, the validity of the principle corresponds to a recent theorem of Komlos [8] which states that from any norm-bounded sequence $F$ in $L^{1}$ a subsequence $F_{0}$ can be so extracted that any arbitrary subsequence $\left\{f_{n}\right\}$ of $F_{0}$ has the property that $\lim _{n \rightarrow \infty}\left(f_{1}+\cdots+f_{n}\right) / n=\alpha$ exists a.e. where $\alpha \in L^{1}$ is the same for all subsequences $\left\{f_{n}\right\}$ of $F_{0}$. (Naturally, $\alpha$ depends on the choice of $F_{0}$.) When $\Pi$ is the Marcinkiewicz generalization of the Kolmogorov theorem to independent identically distributed $X_{n}$ with $\left\|X_{n}\right\|_{p}<\infty, 0<p<2$, I have established the validity of the subsequence principle in [1]. For $\Pi$ of the central limit theorem also, I have shown in [4] in complete detail that the principle in question is perfectly justified. In [2], this and further instances of the principle are discussed. In the present paper my purpose is to announce that the law of the iterated logarithm also falls within the scope of the subsequence principle. More precisely, we have the following theorems.

THEOREM 1. Let $F$ be any norm-bounded (real) sequence in $L^{2}$ over an arbitrary measure space $(\Omega, \Sigma, \mu)$. Then there exist functions $\alpha \in L^{2}$ and

AMS (MOS) subject classifications (1970). Primary 60F15; Secondary 28A65.

Key words and phrases. Subsequence principle, law of the iterated logarithm. 
$\beta \in L_{+}^{2}$ and a subsequence $F_{0}$ of $F$ such that any subsequence $\left\{f_{n}\right\}$ chosen from $F_{0}$ has the following property:

$$
\limsup _{n \rightarrow \infty} \sum_{k=1}^{n}\left(f_{k}-\alpha\right) /(2 n \log \log n)^{1 / 2}=\beta^{1 / 2} \text { a.e. }
$$

THEOREM 2. Let $F$ be any norm-bounded (real) sequence in $L^{\infty}$ over an arbitrary $\sigma$-finite measure space $(\Omega, \Sigma, \mu)$. Then there exist functions $\alpha \in L^{\infty}$ and $\beta \in L_{+}^{\infty}$ and a subsequence $F_{0}$ of $F$ such that for any subsequence $\left\{f_{n}\right\}$ from $F_{0}$ and any sequence of real numbers $\left\{a_{n}\right\}$ such that $A_{n}=\sum_{k=1}^{n} a_{k}^{2} \rightarrow \infty$ as $n \rightarrow \infty$ and $a_{n}=o\left(A_{n} / \log \log A_{n}\right)^{1 / 2}$, the following property is valid:

$$
\limsup _{n \rightarrow \infty} \sum_{k=1}^{n} a_{k}\left(f_{k}-\alpha\right) /\left(2 A_{n} \log \log A_{n}\right)^{1 / 2}=\beta^{1 / 2} \text { a.e. }
$$

It is clear that both the theorems follow in complete generality from the special case where $\mu$ is a probability measure. Indeed this simple remark is applicable to the other cases of the subsequence principle as well. It is to be noted also that our theorems imply the classical law of the iterated logarithms for independent, identically distributed random variables or a little more generally for symmetrically dependent or exchangeable random variables. Theorem 2 generalizes a theorem of Mary Weiss [12] for uniformly bounded orthonormal sequences. However as I have remarked elsewhere [2] it can also be deduced rather easily from the aforementioned theorem of Mary Weiss. Nevertheless my method using martingale theory avoids the cumbersome calculations of [12]. It is to be remarked that no generalization of Theorem 1 allowing for arbitrary coefficients $\left\{a_{n}\right\}$ as in Theorem 2 is possible (cf. [2]). Further these theorems are the best possible in a very definite sense illustrated by known theorems for independent random variables (cf. Hartman and Wintner [6], Strassen [11], Marcinkiewiez and Zygmund [9]).

The detailed proofs will appear elsewhere [5]. The main idea is to obtain a subsequence which behaves like a martingale which when suitably truncated (in the fashion of Hartman and Wintner [6]) becomes amenable to a recent law of the iterated logarithm due to Stout [10] (which itself is a perfect generalization of the classical law of Kolmogorov [7] from which Hartman and Wintner [6] derived their result).

It may be remarked also that the proof of Theorem 2 is quite similar to and considerably easier than that of Theorem 1 . Further, the functions $\alpha$ and $\beta$ occurring in Theorem 2 are $\left(L^{2}\right.$-) weak cluster points of the sequences $\left\{f_{n}\right\}$ and $\left\{\left(f_{n}-\alpha\right)^{2}\right\}$ respectively. The same is true for $\alpha$ in Theorem 1 but in general the relationship of $\beta$ to $\left\{f_{n}^{2}\right\}$ is a bit more complicated. If the sequence $\left\{f_{n}^{2}\right\}$ is weakly sequentially compact in $L^{1}$ then $\beta$ also can be identified as a weak cluster point of $\left\{\left(f_{n}-\alpha\right)^{2}\right\}$. In general, $\beta$ is 
such that for a suitable subsequence $\left\{f_{n(j)}\right\}, N^{-1} \sum_{j=1}^{N}\left(f_{n(j)}-\alpha\right)^{2} \rightarrow \beta$ a.e. Intuitively speaking, $\alpha$ and $\beta$ play roles of mean and variance respectively.

\section{REFERENCES}

1. S. D. Chatterji, A general strong law, Invent. Math. 9 (1969/70), 235-245. MR 42 \#1183.

2. - Un principe de sous-suites dans la théorie des probabilités, Séminaire de probabilités VI, Strasbourg, Lecture Notes in Math., vol. 258, Springer Verlag, Berlin and New York, 1972, pp. 72-89.

3. - Les martingales et leurs applications analytiques. Ecole d'été de probabilités: processus stochastiques (Bretagnolle, Chatterji, Meyer), Lecture Notes in Math., vol. 307, Springer-Verlag, Berlin and New York, 1973.

4. - A principle of subsequences in probability theory.(The central limit theorem), Advances in Math. (to appear).

5. - A principle of subsequences in probability theory. II. (The law of the iterated logarithm) (to appear).

6. P. Hartman and A. Winnter, On the law of the iterated logarithm, Amer. J. Math. 63 (1941), 169-176. MR 2, 228.

7. A. Kolmogoroff, Über das Gesetz des iterierten Logarithmus, Math. Ann. 101 (1929), 126-135.

8. J. Komlós, A generalization of a problem of Steinhaus, Acta Math. Acad. Sci. Hungar. 18 (1967), 217-229. MR 35 \#1071.

9. J. Marcinkiewicz and A. Zygmund, Remarque sur la loi du logarithme itéré, Fund. Math. 29 (1937), 215-222.

10. W. F. Stout, A martingale analogue of Kolmogorov's law of the iterated logarithm, Z. Wahrscheinlichkeitstheorie und Verw. Gebiete 15 (1970), 279-290. MR 45 \#2778.

11. V. Strassen, A converse to the law of the iterated logarithm, $\mathrm{Z}$. Wahrscheinlichkeitstheorie und Verw. Gebiete 4 (1965/66), 265-268. MR 34 \#850.

12. Mary Weiss, On the law of the iterated logarithm for uniformly bounded orthonormal systems, Trans. Amer. Math. Soc. 92 (1959), 531-553. MR 21 \#5844.

Département de Mathématiques, École Polytechnique Fédérale de lausanne, 26, Ave de Cour, 1007 Lausanne, Switzerland 